Revue européenne des sciences sociales

European Journal of Social Sciences

XLIV-134 | 2006

Quel(s) défi(s) pour les sciences sociales à l'heure de la mondialisation?

\title{
La matrice interactive du don
}

André Petitat

\section{OpenEdition}

1 Journals

Édition électronique

URL : http://journals.openedition.org/ress/304

DOI : $10.4000 /$ ress.304

ISSN : 1663-4446

Éditeur

Librairie Droz

Édition imprimée

Date de publication : 1 juin 2006

Pagination : 215-230

ISBN : 9-782-600-01095-5

ISSN : 0048-8046

Référence électronique

André Petitat, "La matrice interactive du don », Revue européenne des sciences sociales [En ligne],

XLIV-134 | 2006, mis en ligne le 15 octobre 2009, consulté le 21 avril 2019. URL : http://

journals.openedition.org/ress/304; DOI : 10.4000/ress.304

(c) Librairie Droz 


\section{André PETITAT}

\section{LA MATRICE INTERACTIVE DU DON}

Dans un hommage à Gérald Berthoud, écrire sur le don c'est d'abord reconnaître son apport dans ce domaine, en précurseur et en animateur du regain d'intérêt pour Marcel Mauss et pour les manifestations oblatives dans le monde moderne'. C'est se mettre en position de contre-don.

Ces quelques pages mobilisent les postures du don pour illustrer la pertinence d'une notion que l'on peut dénommer «matrice interactive». Ce concept désigne la pluralité des postures caractéristiques d'un contexte stéréotypique du genre promesse, rencontre, lutte, poursuite, concours, ordre, demande d'aide, contrat, don, etc. Son intérêt est à la fois théorique et méthodologique.

Deux séries de travaux ont contribué à la proposition de ce concept. La première concerne la genèse de la compréhension de l'action et la seconde la typification des actions et interactions.

\section{SOCIOGENÈSE DE LA COMPRÉHENSION}

Dans l'histoire de l'espèce humaine, complexification de l'action et complexification de la compréhension sont solidaires. Elles forment le cercle ou plutôt la spirale herméneutique de base des relations humaines. Il s'agit d'une sociogenèse au long cours sur laquelle les travaux relatifs à l'ontogenèse de la compréhension chez l'enfant et chez les primates supérieurs jettent une infidèle mais précieuse lumière. La rupture entre comportement et action repose sur la représentation, cette forme première du virtuel. La représentation symbolique - qui prend appui d'abord sur l'image, puis sur le symbole et enfin sur le signe - permet d'opérer un retour sur le comportement appris et de fonder l'action dans un espace de réversibilité qui est en même temps un espace d'indétermination partielle.

La sociologie et l'anthropologie compréhensives ont surtout pris en compte la relation d'altérité et de similitude entre le $J e$ et le $T u$. L'Autre est compréhensible grâce au Nous d'une commune humanité que nous devons reconstruire. Les pages qui suivent insistent sur un autre aspect, tout aussi important, à savoir que la complexification de la compréhension est constitutive de la complexification des relations. La première ne procède pas d'un déroulement de potentialités prédéfinies, pas plus que la seconde ne procède d'un déchiffrage de possibilités inscrites

Voir notamment ses articles dans G. Berthoud et G. Busino, Paroles reçues. Du bon usage des sciences sociales, Genève, Droz, 2000. 
en puissance dans les contextes relationnels. Au cours de sa sociogenèse, l'enfant comprend d'abord le rôle des capacités motrices puis celui des perceptions et enfin celui des croyances aux $1^{\text {er }}, 2^{\mathrm{e}}$ et $3^{\mathrm{e}}$ degrés. A mesure qu'il progresse, l'éventail de ses possibles interactifs s'élargit. La recherche viendra peu à peu préciser les étapes de cette construction compréhensive et documenter sa sensibilité aux variations socioculturelles ${ }^{2}$.

\section{CATÉGORISATION DE L'ACTION}

La seconde série de travaux qui appuie la notion de matrice interactive est relative à la catégorisation spontanée ou savante de l'action et des interactions. Examinons brièvement les apports principaux d'abord en sociologie puis en psychologie et en psychologie sociale.

Max Weber a distingué quatre types d'action, d'après le sens visé par l'acteur: les actions rationnelles en finalité, rationnelles en valeur, affectives et traditionnelles. Dans cette classification, il ne considère pas du tout les variations contextuelles mais uniquement les ressorts possibles des acteurs. L'individualisme méthodologique s'est inscrit dans cette perspective. Après avoir focalisé son attention sur le calcul rationnel et ses limites, il est revenu à un éventail analogue de « raisons ».

Alfred Schutz, en soulignant l'importance des typifications de l'action d'autrui et de soi-même dans les relations sociales, s'oriente davantage dans la prise en compte des contextes. Nous disposons d'un certain nombre d'outils, socialement approuvés pour une bonne part, pour définir ce qui se passe ici ou là. L'idée de mondes sociaux distincts, reprise de William James, esquisse des contextes aux logiques différenciées.

L'interactionnisme symbolique, également sur le versant de la sociologie compréhensive, a approfondi cette voie en explorant empiriquement les relations in situ. L'ethnométhodologie a poussé cet intérêt jusqu'à la description minutieuse des contextes d'interaction, en particulier des interactions verbales, en s'intéressant tout particulièrement aux procédures qui contribuent à la définition des relations.

Sur le versant de la sociologie explicative, les sociologues ont continué sur la voie ouverte par Emile Durkheim, soit l'intériorisation par les agents de dispositions exigées par le fonctionnement contraignant de la société. L'habitus bourdieusien a explicitement pour but de clore le cercle des déterminismes entre les individus et la «structure des rapports de force et de sens entre groupes sociaux ». Ce schéma est susceptible d'être démultiplié indéfiniment, en ouvrant sur d'innombrables dispositions intériorisées, mobilisées à l'occasion par des contextes correspondants. Cet agent pluriel est un agrégat de microdéterminismes (cf. Bernard Lahire).

Ces deux courants explicatifs et compréhensifs ont leurs correspondants en psychologie et en psychosociologie. Ces deux disciplines ont multiplié les

2 A. Petitat, Secret et formes sociales, Paris, PUF, 1998; et aussi «Contes et normativité », in A. Petitat (éd.), Contes : le singulier et l' universel, Lausanne, Payot, 2002, p. 29-54. 
vocables pour désigner les dispositions intériorisées. Selon les auteurs, il est question de compétences ou d'aptitudes motrices, perceptives, verbales, sociales, de traits de personnalité, d'habitudes et surtout de schèmes et de schémas: schèmes cognitifs et affectifs, particuliers ou généraux, plus ou moins durables ou transférables, etc.

Le courant déterministe porte la marque du behaviorisme et se fonde sur des associations entre schèmes et contextes d'action. Burrhus F. Skinner est un bon représentant de cette tendance, qui a énormément pâti du développement du cognitivisme. Les neurosciences ont pris le relais, dans une tout autre direction. Le second courant accorde à l'acteur un rôle créatif dans ses adaptations pratiques et réfléchies à l'environnement naturel et social. Jean Piaget a joué un rôle central dans la définition de ce courant autoconstructiviste, qui s'est ramifié en direction de la psychologie culturelle et de la théorie de l'esprit.

Avec la distance, ces courants sont peut-être plus complémentaires que contradictoires, à condition qu'ils abandonnent leurs ambitions impérialistes et réductrices. Car, dans la perspective d'une sociogenèse de la compréhension, il ne fait pas de doute que l'action symbolique s'est érigée sur la base du comportement appris et celui-ci sur la base du comportement héréditaire. Sans rechercher à tout prix une conciliation irénique, l'hypothèse d'un comportement et d'une action stratifiés, avec maintien et combinaison des strates les plus anciennes avec les strates les plus récentes, semble la plus plausible.

Qu'une part de nos conduites relève du conditionnement paraît difficile à nier. La publicité table lourdement sur des mécanismes associatifs, sans toutefois obtenir toujours ce qu'elle désire. Nous ne sommes qu'en partie des pigeons.

Entre le niveau du conditionnement et celui de l'action clairement réfléchie et anticipée, on trouve deux zones d'action dont l'étude est particulièrement éclairante, celle de l'habitude et celle de l'innovation non consciente d'elle-même.

La psychologie sociale a apporté au sujet de l'habitude des éléments intéressants. Entre le schéma mental et l'action s'intercalent des processus complexes telles que la perception, la cognition et les attributions sociales. Si un acteur dispose de peu d'informations sur un autre, un groupe ou une situation, il aura tendance à utiliser quelques traits saillants qui s'offrent à lui et à les rapporter à ses propres schémas antérieurs. En revanche, lorsque les informations sur l'objet en cause sont nombreuses, on observe un travail d'intégration des données, en particulier si l'acteur est bien motivé, surtout s'il doit faire attention aux conséquences de son évaluation. Selon Susan T. Fiske et Steven L. Neuberg³ ${ }^{3}$ l'idée qu'on se fait d'un objet social s'inscrit sur un continuum allant des schémas préconçus à un traitement attentif et nouveau des données. En cas d'interaction soutenue avec autrui, on observe une certaine paresse de l'acteur, qui tend à retenir les informations qui confirment l'opinion qu'il s'est faite; la personne observée peut ellemême contribuer à la vérification du jugement de l'observateur (self-fulfilling

S. T. Fiske, S. L. Neuberg, «A continuum of impression formation from category-based to individuating processes», in N. Zanna (ed.), Advances in experimental social psychology, Orlando, Academic Press, vol. 23, 1990, p. 1-74. Voir aussi M. A. Pavelchak, «Piecemeal and category-based evaluation: an idiographic analysis », Journal of Personality and Social Psychology, 56, 1989, p. 354-363. 
prophecy). Mais l'inverse est vrai aussi: nous pouvons agir afin de modifier l'opinion plus ou moins cristallisée d'un observateur ${ }^{4}$. En fin de compte, tant l'observateur que l'observé sont des personnes actives au cours de la perception, du traitement de l'information et sans aucun doute du choix des modalités d'une éventuelle action ou interaction. Si certaines situations et interactions favorisent le maintien des schémas acquis, d'autres invitent à leur dépassement. Même si l'acteur se contente de stimuli sommaires pour porter son jugement en activant ses schémas établis, il ne se situe pas dans le registre du conditionnement mais dans celui de l'action conduite avec une certaine passivité perceptive et cognitive, passivité dont quantité d'éléments peuvent le faire sortir à tout moment.

Les travaux sur les attributions mettent aussi en évidence l'importance du travail cognitif dans l'évaluation de la situation et le déploiement de l'action et nous éloignent un peu plus encore du schéma Stimulus - Réponse et de la pertinence d'un tel schéma, même assoupli, pour examiner les habitudes ou régularités actionnelles.

Il existe un autre registre de l'action pratique à distinguer de celui de l'habitude. Ce registre varie en fonction de la genèse de la compréhension de l'action; il correspond à la zone de pointe, celle qui ne pourra être pensée explicitement qu'à partir d'une étape métacompréhensive à venir. Edouard Claparède et Jean Piaget ont apporté sur ce point des éclairages encore valables aujourd'hui. Nos habiletés cognitives nouvelles se manifestent d'abord pratiquement avant d'être réfléchies. Cette constatation, bien établie depuis maintenant environ un siècle en psychologie, n'est pas valable seulement dans le cadre de la progression métacognitive. Beaucoup de nos transformations relationnelles - qui ne présupposent pas de saut cognitif particulier - interviennent en catimini, au plan des pratiques, avant de faire l'objet d'un travail réflexif explicite. Remarquons que nous sommes en ce cas à l'opposé de l'habitude et de la paresse cognitives; il faudrait plutôt parler de réflexivité pratique.

En résumé, les travaux de la psychologie sociale et de la psychologie invitent à ne pas surestimer le modèle du conditionnement, peu pertinent pour les domaines des habitudes et des pratiques innovantes pas ou peu conscientes d'elles-mêmes. Les critiques envers l'intellectualisme de l'action rationnelle, justifiées en cas de réduction vers le haut, ne sauraient légitimer une réduction vers le bas. Pour saisir les dynamiques de production et de reproduction du social, la sociologie n'a pas d'autres choix que de prendre en compte les processus de perception, de cognition, d'émotion, de mémorisation, de catégorisation et d'attribution sociales. Ces processus mettent l'accent sur l'activité des sujets en interaction. Ils apportent de l'eau au moulin du modèle adaptatif et constructiviste.

\section{SCHÉMAS PLURIELS OU MATRICES INTERACTIVES}

La notion de matrice interactive considère la complexité relationnelle comme le produit de deux sources fondamentales: la compréhension du sujet et le contexte

4 W. B. Swann, «Identity negociation: where two roads meet», Journal of Personality and Social Psychology, 53, 1987, p. 1038-1051. 
dans laquelle elle s'insère. Nul doute que ces deux réalités soient inépuisables et leur rencontre plus encore. Cette inépuisabilité n'empêche pas que nous dessinions des modèles simplifiés des deux dimensions pour tenter ensuite de les combiner.

Nos capacités compréhensives nous permettent de distinguer ce qui relève de la contingence (rencontre imprévisible), de la causalité naturelle (une avalanche se déclenche), du comportement non réflexif (le chat mange les souris) ou de l'action. Cette dernière se subdivise en action non stratégique (plaisir moteur, perceptif, imaginaire, esthétique), involontaire (maladresse, erreur, confusion, etc.) ou stratégique. Celle-ci à son tour comprend deux catégories fondamentales: l'action orientée vers la nature ou l'action orientée vers autrui. Cette dernière catégorie est de loin la plus importante. Les efforts des sociologues considèrent les moyens et les fins dans les rapports à autrui. Les efforts des psychologues, d'abord fascinés par les rapports à la nature, s'orientent de plus en plus vers les rapports à autrui. L'approche constructiviste fournit pour sa part des points de repère identifiés comme importants dans la sociogenèse de la compréhension: rôle de la force, rôle de la perception et rôle des croyances dans l'action. La compréhension des croyances au premier degré donne notamment accès au mensonge, celle au deuxième degré ouvre sur la règle commune et celle au troisième degré rend possible l'intégration de systèmes simples dans des systèmes complexes.

L'événement, le comportement et l'action sont des opérateurs de transformation. Ils peuvent se combiner. Insérés dans un contexte, ils donnent lieu à une opération qui peut éventuellement, à son tour, servir d'opérateur dans une transformation plus vaste, et ainsi de suite. C'est ici qu'intervient le lien avec le récit. Le récit, qu'il soit factuel ou fictionnel, met en scène une série de transformations que l'auteur fait survenir et que le lecteur comprend parce que tous deux maîtrisent de tels opérateurs et savent les mettre en contexte.

Ma principale source d'inspiration relative à la modélisation des contextes provient de l'analyse de trois importants corpus de contes de tradition orale: des contes formulaires ou contes sériels (corpus intégral des contes-types de la classification Aarne-Thompson), des contes d'animaux (corpus intégral des résumés des contes-types Aarne-Thompson) et des contes ordinaires (une centaine de contes de Grimm $)^{5}$. La narration modélise; elle met par conséquent à notre disposition la façon dont auteurs et lecteurs découpent les contextes d'interaction et les stylisent. Ce travail de modélisation simplificatrice a été à l'œuvre durant des millénaires dans la tradition orale, et on peut estimer que les contes nous offrent une palette large et variée de la stéréotypisation des contextes interactifs, et partant de leur connaissance ordinaire ${ }^{6}$.

La variété infinie des contextes n'exclut pas le retour d'un certain nombre de situations interactives stéréotypiques, déjà évoquées en début d'article (interdic-

A. Petitat, R. Baroni, «Dynamique du récit et théorie de l'action», Poétique, $\mathrm{n}^{\circ}$ 123, 2000, p. 353-379; A. Petitat, S. Pahud, «L'interaction narrative et ses surprises: contes-attrapes et autres curiosités de la littérature orale », Poétique, n 138, 2004, p. 159-181.

6 Je présente ici la distinction entre compréhension et contexte comme si elle était évidente, mais c'est loin d'être le cas puisque, comme nous l'avons vu, la complexité de la compréhension et des interactions varie de façon concomitante. 
tion, serment, rencontre, contrat, etc.), présentes quelle que soit l'aire culturelle d'appartenance des contes. Il est impossible d'étudier ici chacune des matrices interactives qui résultent du croisement d'une interaction stéréotypique et des opérateurs de transformation, en dessinant chaque fois un nœud relationnel aux multiples issues. Il est préférable de se concentrer sur un seul exemple, celui du don. L'inventaire des possibles de ce nœud interactif a été conduit à partir de l'analyse de nos trois corpus. Un tel inventaire n'a bien sûr rien d'exhaustif puisqu'il est relatif aux modèles narratifs du don réalisés par le conteur populaire traditionnel. On pourrait par exemple le compléter par une analyse du don dans les relations amicales, amoureuses ou familiales.

\section{LE DON ET LA CIRCULATION DES «OBJETS»}

La circulation des « objets » prend trois formes génériques principales, celle du don unilatéral, celle du don réciproque et celle de l'échange négocié (troc et échange monétaire). La réciprocité oblative s'oppose à l'équivalence contractuelle; la première implique un non-dit sur la nature et les délais du «rendre», tandis que la seconde suppose au contraire un accord en principe transparent sur la nature de la transaction. L'unilatéralité du don est irréductible aussi bien à la réciprocité qu'à l'équivalence, qu'elle permet de relativiser et de penser.

Les trois formes génériques de la circulation s'appliquent à des «objets » très divers, parmi lesquels nous pouvons distinguer principalement les biens (choses, végétaux, animaux, esclaves) et les services (aide, travail), l'influence, les sentiments et les savoirs. La plupart des recherches se sont concentrées sur les biens et les services.

Enfin, last but not least, unilatéralité, réciprocité et équivalence connaissent des applications aussi bien positives que moralement douteuses ou condamnables. On peut, gratuitement, faire un compliment ou une attaque, sauver la vie ou donner la mort. Si la réciprocité est souvent positive, l'ingratitude voire la trahison ne sont pas rares. Enfin, le contrat est fréquemment l'occasion de tromperies élaborées. Je parlerai par la suite d'unilatéralité négative et de réciprocité négative?.

\section{LE DON CONTEMPORAIN \\ ET LA NOSTALGIE DE LA BONNE COMMUNAUTÉ}

Marcel Mauss s'est appliqué à séculariser les représentations religieuses du don. Entre unilatéralité et équivalence, il a tracé le territoire du don réciproque, à la fois libre et obligatoire, gratuit et intéressé, source de paix opposée à la guerre. A mi-chemin entre ciel et terre, le don maussien est une sorte de forme intermédiaire entre l'échange réputé froid et intéressé et le don décrit comme chaleureux et gratuit. Une certaine nostalgie du communautaire remplace la métaphysique

A. Petitat, «Le don: espace imaginaire normatif et secret des acteurs», Anthropologie et sociétés, $\mathrm{n}^{\circ} 19,1995$, p. 17-44. 
oblative. Le don réciproque devient porteur de lien positif, rédempteur d'une société gangrenée par l'inflation des choses, des contrats, de l'égoïsme et des bureaucraties, qui privilégie «l'être en apesanteur sociale».

Cette idéalisation du don des communautaristes correspond à l'idéalisation du contrat des libertariens. En réalité, ces formes stéréotypiques de la circulation des «objets » comportent toutes deux des zones d'ombre peu paradisiaques. Ni le don ni le contrat ne sont réductibles à leurs idéaux asymptotiques. Ces formes de base des échanges sont toutes deux travaillées par des virtualités nettement moins rassurantes de nos espaces de jeu. Une matrice interactive est un outil qui tente de rendre compte de la plus grande diversité des postures possibles attachées à un contexte relationnel et non seulement de celles qui entrent en résonance avec un engagement de valeur.

\section{DON ET NIVEAUX DE COMPRÉHENSION DE L'ACTION}

Le don unilatéral est probablement la forme la plus primitive de circulation des objets. Il dérive vraisemblablement des comportements nourriciers, affectifs et protecteurs des parents à l'égard de leur progéniture. Nous connaissons peu de choses sur cette transition de la nature à la culture, plus primitive probablement que l'organisation exogamique de la famille. La sociogenèse de l'enfant indique que ce dernier comprend initialement le recevoir avant le donner et le rendre. Les premiers éléments de réciprocité concernent à l'évidence les affects, avant les biens. Ce qui est à moi et à toi fait ensuite l'objet d'une construction sans doute fortement marquée par le contexte social. La compréhension des capacités sensori-motrices dans l'action amène à de précoces et délibérés rapports de coopération et de force, suivis des ruses perceptives. La réciprocité affective et sensori-motrice, positive ou négative, ne peut s'installer qu'à la hauteur assez primitive de cette compréhension de l'action. Mais tout porte à croire qu'elle existe déjà à ce niveau ${ }^{8}$.

La compréhension du rôle des croyances dans l'action enrichit considérablement la complexité relationnelle. Conclure un contrat implique une croyance réciproque dans les modalités convenues d'une transaction. La réciprocité attendue mais non exprimée, avec un décalage temporel parfois important entre don et contre-don, suppose également que les partenaires se réfèrent à des principes communs de conduite difficiles à contextualiser adéquatement. La régulation des principes qui président à ces échanges se complexifie elle-même jusqu'à l'âge adulte.

8 Les travaux remarquables de F. de Waal indiquent qu'elle est déjà présente chez les chimpanzés, sous trois formes: une première fondée sur l'affection et le soutien réciproque durable (entraide, soutien lors des combats, sans avoir besoin de mémoriser les faveurs données et reçues); une deuxième fondée sur la réciprocité immédiate (ex.: un chimpanzé partage sa nourriture avec un autre qui vient de l'aider à l'obtenir); une troisième enfin fondée sur une mémoire plus longue des prestations, où les individus se souviennent des bienfaits reçus lorsqu'il est question de favoriser tel ou tel membre du groupe (ex.: un chimpanzé donne préférentiellement de la nourriture à un autre qui l'a quelque temps auparavant aidé à s'épouiller). Cf. F. de Waal, «Le commerce chez les animaux », Pour la science, $\mathrm{n}^{\circ} 331,2005$, p. 34-41. 


\section{DON UNILATÉRAL ET CONTES DE TRADITION ORALE}

Les genres de la tradition orale ne sont pas complètement étrangers à la complexification de l'action. Les contes formulaires ou sériels, les contes d'animaux et les contes merveilleux présentent un ordre de complexité croissante, au plan de la structure narrative comme au plan de l'action fictionnelle. Les premiers, à quelques exceptions près, sont visiblement destinés aux tout petits; les deuxièmes s'intéressent principalement à la transgression des régularités et des règles, alors que les troisièmes ajoutent le plus souvent l'étage de la régulation.

\section{L'unilatéralité positive}

Elle est présente dans nos trois corpus de contes, mais surtout dans les contes formulaires et les contes ordinaires. Les contes d'animaux, axés majoritairement sur l'exploration des ruses, ne la mettent en scène que marginalement.

Parmi les contes formulaires, prenons en exemple une variante allemande du conte Et j' allai chez mon frère. Douze fois de suite (pour les douze jours de Noël), un enfant (à la première personne) va chez son frère et reçoit à chaque fois des cadeaux cumulatifs de plus en plus importants: deux poules, puis trois oies et deux poules, puis quatre cochons, trois oies et deux poules. Les onzième et douzième jours se signalent par une véritable orgie de cadeaux invraisemblables: églises, autels, prêtres, servantes, bébés, etc.

Ce conte est construit selon une double répétition. La première se rapporte aux visites successives durant les douze jours de Noël. La seconde concerne les cadeaux reçus et indique clairement que tout est susceptible de se muer en don, jusqu'aux personnes elles-mêmes (frontière sacrée ignorée). La relation du petit frère au grand frère ajoute une dimension oblative aux objets, dimension étendue à profusion dans tous les sens.

Passons directement aux contes merveilleux. Les circonstances dans lesquelles interviennent les dons unilatéraux positifs sont très diversifiées. Esquissons une classification sous l'angle de la démarche du donateur:

1) le donateur merveilleux, qui connaît à distance les besoins du receveur sans même que celui-ci les ait exprimés, propose spontanément une aide (ex. L'enfant de Marie: la Vierge Marie, sachant qu'un pauvre bûcheron n'arrive pas à nourrir sa fille, lui propose d'élever celle-ci au paradis);

2) le donateur réagit spontanément après la constatation d'un besoin (dans Les trois feuilles du serpent, le fidèle serviteur repêche son maître qui a été jeté à l'eau et le ressuscite grâce aux trois feuilles);

3) l'information elle-même sur ce qui se passe prend la forme d'un don unilatéral (dans Cendrillon, deux colombes parlent suffisamment fort pour que le roi entende et réalise qu'il est dupe; dans L'énigme, la fille informe discrètement les hôtes qu'il ne faut rien manger car sa mère est une dangereuse sorcière);

4) le donateur réagit à une situation jugée anormale qu'il explore afin d'identifier le problème, indépendamment du receveur (dans Le Petit Chaperon rouge, le chasseur entend des ronflements inhabituels et entre dans la maison pour voir ce qui se passe); 
5) le donateur réagit à une situation jugée anormale, en demandant au receveur de s'exprimer sur ce qui se passe (cf. Les trois fileuses : la reine en passant entend des cris et des gémissements, entre dans la maison et demande à la mère de s'expliquer);

6) le donateur demande d'entrée de jeu au récepteur de formuler ses désirs (cf. la grosse grenouille dans Les trois plumes);

7) le donateur répond à une demande explicite (dans Cendrillon, à la demande de l'héroïne, les oiseaux viennent l'aider à trier les lentilles et l'oiseau blanc fournit une tenue somptueuse). La façon de formuler la demande peut être plaisante et flatteuse, comme dans Jeannot et Margot: «Canard blanc, canard blanc / Ici Margot et Petit-Jean / Aucun sentier et pas de pont / Porte-nous sur ton beau dos rond $»$;

8) Dans certains cas, un vœu est formulé sans s'adresser à quiconque en particulier. Ce vœu est alors satisfait soit par un donateur particulier soit sans qu'aucun donateur apparaisse. C'est ainsi que le héros de La sacoche, le vieux chapeau et la petite trompette souhaite vivement pouvoir manger alors qu'il est affamé; aussitôt apparaît une table recouverte d'une petite «nappe-couvretoi » et chargée de mets succulents.

Cette liste met en exergue la nature de la démarche du donateur, qui oscille entre pure spontanéité et réaction à une demande explicite. Cette dimension n'est évidemment pas la seule à donner sa tonalité particulière à l'unilatéralité. Il y a également la nature du donateur (divinité, personnage merveilleux, personnage ordinaire, humain, animal ou autre) et surtout la relation entre donateur et receveur (se connaissent ou ne se connaissent pas, sont liés par une solidarité familiale, amicale, religieuse, etc.).

\section{L'unilatéralité négative}

Elle est rare dans les contes merveilleux, pour la simple raison que les mauvaises actions y sont généralement punies, ce qui introduit une réciprocité négative. Peu présent ne veut pas dire absent. Dans la version des frères Grimm, la punition de la terrible sorcière de Raiponce est oubliée, l'épilogue ne faisant état que du bonheur des amants réunis. La sorcière de L'énigme, qui a tenté d'empoisonner ses hôtes, n'est pas inquiétée non plus. Selon un schéma analogue, les mauvaises marâtres ne sont pas toujours sanctionnées. Par exemple, le conte Cendrillon punit les deux odieuses demi-sœurs, mais pas leur mère pourtant tout aussi détestable. La même situation survient dans Dame Holle. Notons aussi que l'unilatéralité négative se drape parfois du manteau de la positivité: on se souvient de l'orgueilleuse reine déguisée en colporteuse qui offre une pomme empoisonnée à Blanche-Neige. Notons enfin que l'unilatéralité négative peut survenir sur la base d'un simple vœu qui ne s'adresse à personne en particulier. Dans Les sept corbeaux, le père s'emporte contre ses enfants qui tardent à rapporter de l'eau et dit: «Je voudrais les voir tous transformés en corbeaux!». Aussitôt il entend un froissement d'ailes et voit sept corbeaux s'éloigner.

Les contes d'animaux comportent souvent le couple action - réaction. Le rusé renard accomplit-il des actions pendables, sa victime tentera souvent de se venger, 
que ce soit avec ou sans succès. Nous retrouverons ces contes au volet suivant de la réciprocité. Ceci dit, le don négatif unilatéral est très fréquent dans les contes d'animaux. Dans le premier conte-type d'animaux de la classification AarneThompson, Le vol de poissons, le renard affamé qui fait le mort est chargé par le pêcheur sur sa charrette et il profite de son inattention pour la vider de ses poissons. Non seulement le voleur reste impuni, mais sa victime se fait en plus houspiller par sa femme. Les mauvais conseils donnés par le rusé au stupide forment une autre catégorie de dons négatifs. On y trouve La pêche à la queue: le renard fait croire au loup qu'il a pêché les poissons qu'il est en train de dévorer en laissant tremper sa queue dans un des trous fait dans la glace par les pêcheurs. Le loup s'exécute, l'eau gèle, les pêcheurs battent le loup. Celui-ci parvient finalement à se libérer, mais en y laissant sa queue. Certains conseils conduisent à la mort des dupes. Voici encore quelques leviers du don négatif: susciter une peur panique qui engendre des désagréments, jusqu'à la mort; provoquer par des agacements répétés un énervement fatal; défier un adversaire pour l'amener à tenter une action impossible qui se retourne contre lui; amener les autres à s'entretuer; prétendre rendre un service alors que le but est de faire du mal; tuer le partenaire qui dévoile la trahison d'une entente; induire une fausse croyance pour susciter une action dangereuse voire mortelle pour celui qui l'exécute; susciter par des mensonges un comportement altruiste en sa faveur; se débarrasser de son ennemi en conseillant au roi malade de s'enrouler dans sa peau pour guérir; battre un juge gênant; accuser faussement pour légitimer une prédation, etc.

Dans les contes formulaires, certaines chaînes d'accusation peuvent être mises dans la catégorie de l'unilatéralité négative (A accuse $\mathrm{B}$ qui accuse $\mathrm{C}, \ldots$ jusqu'à un bouc émissaire final); certaines chaînes d'accidents également (A heurte involontairement $\mathrm{B}$ qui tombe sur $\mathrm{C}$, etc.), bien qu'ici les désagréments occasionnés à autrui surviennent de manière involontaire; les séries de prédation sans réaction également (X mange des quantités astronomiques de choses, d'animaux et de personnes). Dans le conte La fille abandonnée dans l'arbre par ses sœurs, la malheureuse lance des appels à l'aide ignorés par quatre passants successifs; finalement une panthère passe et la dévore.

\section{DON RÉCIPROQUE ET CONTES DE TRADITION ORALE}

Quatre principaux cas de figure se présentent: la réciprocité positive, la réciprocité négative, la réciprocité positive-négative et la réciprocité négative-positive. D'une manière générale, quel que soit le type de récit, la réciprocité est plus fréquente que l'unilatéralité. Elle est porteuse de continuité actionnelle dans le récit. Un méfait initial peut être puni à la toute fin, venant ainsi clore une tension narrative ouverte dès le début.

\section{La réciprocité positive}

Ce type d'échange est très courant dans les contes merveilleux; il se manifeste surtout lors de l'aide dont bénéficie le héros, plus rarement lors de sa récompense finale. Le canevas classique de la princesse promise contre l'extermination d'un 
monstre relève plus du contrat que du don puisque prestation et contre-prestation sont fixées à l'avance. Mais il arrive que la récompense relève du contre-don, comme dans La reine des abeilles.

Les soutiens dont bénéficient le héros dépendent assez souvent d'un ou de plusieurs dons (souvent trois) réalisés sans souci d'un retour, donc dans un esprit d'unilatéralité, qui appelle(nt) ultérieurement un ou plusieurs contre-dons lors de moments décisifs. Dans L'oie d'or, le Bêta partage son repas avec un gnome qui se plaint de la faim, ce qui lui vaut par la suite trois interventions magiques capitales. Dans Le serpent blanc, trois dons successifs à des poissons, des fourmis et des corbeaux entraînent trois aides vitales. On trouve dans L'oiseau d'or une contre-partie positive quelque peu surprenante, puisque l'adjuvant magique, un renard, demande au héros de lui couper la tête et les pattes. Le héros refuse ce qu'il perçoit comme le comble de l'ingratitude. Il n'obtempère que devant l'insistance de son bienfaiteur ... et le renard se transforme en prince et futur beau-frère.

Le pêcheur et sa femme propose une réflexion sur la réciprocité. Un pêcheur attrape un turbot qui déclare être un prince ensorcelé et demande d'être remis à l'eau. Demande immédiatement acceptée (don unilatéral). La femme du pêcheur persuade son homme d'aller réclamer en contre-partie une jolie chaumière à la place de son vieux «pisse-pot». Contre-don accordé. Bientôt insatisfaite, elle réclame des contre-dons chaque fois plus importants: un château, puis les statuts de reine, d'impératrice, de papesse et finalement de Dieu. Cette dernière requête lui vaut de retourner à la case départ, dans son «pisse-pot».

Une minorité de contes d'animaux illustrent la réciprocité positive. C'est le cas de L'abeille tombe dans l' eau : l'abeille en train de se noyer est sauvée par la colombe. Plus tard l'abeille pique un chasseur qui s'apprête à tuer la colombe. L'épine arrachée de la patte du lion propose un schéma semblable, avec un lion reconnaissant envers son bienfaiteur.

Les contes formulaires ignorent presque complètement la réciprocité positive. Le récit Comment l'homme riche paya son valet est une exception qui se situe entre don et contrat: le maître donne à son valet une poule la première année, un coq la seconde, puis une oie, une chèvre, une vache, un cheval, sa fille et finalement sa ferme.

\section{La réciprocité négative}

Du côté des contes merveilleux, Dame Holle et Les trois petits hommes de la forêt occupent une position transitoire entre réciprocités positive et négative puisqu'ils opposent l'une à l'autre, comme s'ils voulaient administrer une leçon de morale. Une orpheline harcelée par sa marâtre et sa fille rend des services appréciables à Dame Holle, qui fait tomber sur elle une pluie d'or. Sa demi-sœur, envieuse, tente d'obtenir la même récompense, mais elle est tellement peu respectueuse et fainéante qu'elle reçoit une pluie de poix dont elle ne pourra jamais se débarrasser.

Cette dernière logique est caractéristique des contes qui comportent un méfait initial finalement puni. Ainsi, dans L'os chanteur, l'aîné tue son frère, s'empare du trophée et épouse la princesse promise; toutefois, un os du cadet utilisé comme embouchure d'une corne d'appel dénonce le forfait; le fratricide est cousu dans un sac et jeté à l'eau. Dans Le diable et ses trois cheveux d'or, le roi essaie à plusieurs 
reprises d'attenter à la vie d'un jeune homme auquel on a prédit qu'il épouserait la fille du roi; finalement, il est condamné à faire éternellement le passeur sur une rivière.

La réciprocité négative s'applique parfois au niveau des adjuvants potentiels du héros. Ainsi, dans L'oie d'or, les deux frères qui refusent de partager leur repas avec un gnome affamé, futur bienfaiteur de leur cadet, se blessent peu après.

La réciprocité négative est fortement présente dans les contes d'animaux. On se souvient bien sûr du Loup et des sept chevreaux: le loup mange d'abord les chevreaux, mais ensuite Biquette lui ouvre le ventre, sauve ses petits et remplit la panse avec des cailloux; le loup se noie. Dans Le chat, le coq et le renard vivent ensemble, le renard emporte le coq en l'absence du chat. Le chat sauve le coq et tue les petits du renard. Dans Le renard mange la viande du puma et s' endort, la réaction du puma ne se fait pas attendre: dès qu'il se réveille, il dévore le renard. Dans Aveugler le garde, un lapin est fait prisonnier; pour s'évader, il crache du jus de tabac dans les yeux du garde.

Les contes formulaires comportent également des éléments de réciprocité négative. Dans J'ai tué ma grand-mère, un petit-fils tue son aïeule parce qu'elle a refusé de lui cuire un lapin; il tue ensuite un prêtre qui le blâme et obtient d'un moine l'absolution en le menaçant de mort! Le conte L'oiseau indifférent à la douleur débute par un oiseau qui mange des mangues; l'homme le frappe contre les racines du manguier, le met dans l'eau, le frappe par terre, contre un échalas, un cadre de porte, lui brûle les plumes, etc., jusqu'à ce que finalement l'oiseau pique sur le nez de l'homme et que celui-ci en meure.

\section{La réciprocité trahie: don positif suivi d'un contre-don négatif}

Cette succession inattendue de postures inverse complètement la logique de la réciprocité (et appelle d'ailleurs la plupart du temps une punition). Dans L'oiseau d'or, les deux frères aînés libérés par leur cadet poussent ce dernier dans un puits, lui volent ses trophées et se présentent en héros à la cour. Dans Les deux compagnons, le méchant, qui a bénéficié de la générosité du premier et de sa joyeuse compagnie, lui arrache un œil puis l'autre en échange de nourriture dans une situation critique.

Ce genre de contrastes est aussi présent dans les contes d'animaux. Deux contes attirent en particulier l'attention par leur ressemblance. Dans Le garçon et l'ours dans le trou, le premier personnage aide le second à sortir du trou; une fois tiré d'affaire, l'ours aide à son tour le garçon. Nous sommes dans la réciprocité positive. Dans le second conte, nous avons également deux personnages dans une fosse. Le renard grimpe sur le loup et parvient à sortir; hors d'affaire, il souhaite à son compagnon de crever de faim et s'en va. L'insuffisance de réciprocité suffit à faire problème: dans Le conseil de la grenouille, un homme prend soin d'une grenouille pendant l'hiver. Au printemps, la grenouille lui donne un conseil. Ce n'est pas assez pour le sauveteur, qui bat la grenouille. C'est pourquoi la grenouille est bossue. Dans Le serpent ingrat remis en captivité, un homme secourt un serpent qui, en retour, tente de tuer son sauveur. Le renard conseille à l'homme de remettre le serpent en captivité.

La réciprocité trahie existe aussi dans les contes formulaires. Une Poursuite cumulative a pour point de départ des insultes proférées à l'égard de quelqu'un qui 
vient d'offrir son aide. La solution du lapin (Le lapin emprunte de l'argent) pour ne pas rendre l'argent emprunté à un scarabée, un poulet, un renard, un chien et un tigre est ingénieuse: il les fait se manger par ordre croissant. Le chat bien nourri avale sa maîtresse et un tas d'autres choses (Le gros chat). Dans Le corbeau et le pou concluent un pacte d'amitié, ce qui est une promesse de réciprocité positive est aussitôt trahie par le pou qui mange son ami!

\section{La réciprocité-martyre et la réciprocité-pardon}

Nos trois corpus n'offrent pas vraiment d'exemples de l'asymétrie inverse de la précédente, celle où l'on répond par un contre-don positif à un coup, un vol, un sentiment haineux, au mépris, etc. La première partie de Cendrillon n'est pas loin de ce schéma puisque l'héroïne endure son calvaire en restant dans une posture d'humilité souffrante. C'est sa mère qui, à travers le noisetier planté sur sa tombe et l'oiseau blanc qui l'habite, lui procurera une revanche... qu'elle ne refusera pas. Les enfants persécutés par leur marâtre, parce qu'ils sont à sa merci, comme dans Frérot et Sceurette, n'ont souvent pas d'autre choix que de fuir ou de souffrir, l'occasion d'une punition ne venant que plus tard. Dans ces deux cas, la contrainte est telle qu'elle interdit de parler de contre-prestation positive délibérée. Remarquons que les contes chrétiens du recueil des frères Grimm fréquentent surtout les postures de la récompense et de la punition et peu celle de la souffrance rédemptrice ou du pardon. La Vierge Marie pardonne, mais après avoir durement puni pour amener à la repentance (L'enfant de Marie). La Mort marraine paraît plus généreuse car elle pardonne à son filleul une première tromperie importante. Dans L'eau de vie, le roi, après beaucoup de temps, pardonne à son cadet une faute qu'en réalité il n'a pas commise, ce qu'il découvrira bientôt.

C'est peu, et il faut ajouter que les occurrences de ces postures du pardon et du martyre sont encore plus rares dans les contes d'animaux. Parfois le loup et le renard font la paix, mais c'est pour mieux relancer aussitôt les ruses mensongères! Quant aux contes formulaires, aucun ne se rapproche de ces postures.

\section{Mélange de postures}

La trame de fond de nombreux contes est construite autour de la réciprocité et de sa régulation, avec la mobilisation de plusieurs postures différentes. Je n'en cite qu'un exemple, Le vieux Sultan, tiré des contes de Grimm. Un vieux chien a servi un fermier avec une fidélité sans faille (don + ). Son maître le jugeant incapable de continuer à rendre les services qu'il attend de lui projette de le tuer (don-). Le chien, qui a surpris une conversation à ce sujet, va demander conseil à son ami le loup, qui lui suggère un plan ingénieux (don +) : il fera semblant d'emporter l'enfant du fermier et le chien interviendra en sauveteur. Le plan réussit. Le chien est crédité d'un exploit (pseudo-don +), ce qui lui assure la vie sauve et une retraite paisible $($ don + ). Le loup lui demande en contrepartie de fermer un œil s'il vient à prendre un mouton à son maître. Le chien refuse (don -). Lorsqu'il apprend que son ami s'apprête à passer à l'acte, il avertit son maître (don + au maître, don - au loup); le loup manque d'y laisser sa peau (don -). Il provoque le chien en duel pour régler ce qu'il estime être une «affaire d'honneur». Pensant que le chien vient avec un témoin armé d'une épée (erreur de perception), le loup 
se sauve (la régulation de l'absence de réciprocité échoue). Il est ensuite tellement honteux de sa couardise qu'il accepte de faire la paix avec le chien.

\section{CONCLUSION}

Esquissons de façon schématique la matrice du don unilatéral telle qu'elle est suggérée par les typifications proposées dans nos trois types de contes. Elle est relativement simple puisqu'il s'agit d'une action à sens unique, sans le «rendre» de la trilogie de Mauss. Elle comporte trois moments distincts, écartelés entre un pôle positif et un pôle négatif. Dans le pôle positif, les trois moments sont les suivants:

1) Identification d'un besoin chez autrui: dans nos contes, le don unilatéral positif survient toujours après le constat d'un besoin chez autrui par le donateur ou après son expression par le futur receveur; on peut fort bien toutefois imaginer qu'un don unilatéral soit accordé sans égard pour un quelconque besoin. En règle générale, le besoin est correctement cerné, mais il arrive aussi que le donateur se trompe ou soit induit en erreur par une mise en scène ou une fausse déclaration.

2) Offre et réalisation du don: le don est généralement accepté, mais la possibilité d'un refus est toujours possible; il peut être accompagné d'une condition qui limite son usage. L'éventail des dons est très large: biens, nourriture, influence, affection, fidélité, information, etc.

3) Réception: elle est souvent accompagnée d'une expression de reconnaissance; parfois le don est accepté comme s'il était dans l'ordre naturel des choses, sans même un remerciement.

Le pôle négatif présente trois moments opposés:

1) Besoins propres: le malfaiteur est centré sur lui-même; il obéit à ses propres besoins, à ses impulsions ou à sa malveillance. La faim, la cupidité, la jalousie, l'envie, la méchanceté gratuite, le pouvoir, etc., sont ses motivations principales.

2) Méfait: l'éventail est très large et comporte le vol, le malin conseil, la fausse information vitale, la trahison, la diffamation, le mépris, l'exploitation, le harcèlement, l'humiliation, les coups, l'emprisonnement, les blessures, le meurtre, etc. Les usages intempestifs de la force, de la ruse, du mensonge et du non-dit sont au rendez-vous.

3) Réception: dans l'unilatéralité négative, les méfaits sont subis sans réaction régulatrice. Dans les contes d'animaux, plus rarement dans les contes merveilleux, ils servent parfois à faire l'éloge de la ruse et de la réussite.

La matrice du don réciproque est nettement plus compliquée et sa typification fera l'objet d'une autre publication. La réciprocité positive introduit une norme du «rendre » interprétée en général de façon généreuse. Ce n'est toutefois pas toujours le cas; entre générosité et pingrerie, il y a place pour toutes sortes de calculs et de 
tricheries qui font en général l'objet d'évaluation et éventuellement de punition. Dans la réciprocité négative, le «rendre» se présente le plus souvent comme une action régulatrice d'un méfait initial. La réciprocité trahie, la réciprocité-martyre et la réciprocité-pardon occupent les confins d'un espace normatif peuplé de possibles inquiétants ou rédempteurs.

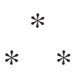

Auteurs et lecteurs configurent et reconfigurent en les modélisant des nœuds interactionnels. Ce travail de typification fictionnel prend beaucoup de liberté avec la réalité quotidienne. Fiction et réalité forment deux mondes distincts qui entretiennent des rapports complexes d'autonomie et de dépendance réciproques. Impossible donc de prendre appui sur la fiction pour en tirer des arguments relatifs à la réalité sociale.

Ceci dit, la lecture de nos différents contes légitime pleinement la caractérisation du don comme «phénomène social total». Biens, services, sentiments, influences et savoirs sont tour à tour incorporés dans deux formes interactionnelles fondamentales, celle du don unilatéral et celle du don réciproque. Dans cette dernière, différents «objets» incommensurables entrent dans des rapports non chiffrables, inexprimables dans une quelconque traduction stable de valeur. L'hétérogénéité irréductible des «objets» semble assurer une approximation et aussi une variabilité qui défient une structuration durable et généralisée de la réciprocité, même dans un univers aussi stéréotypique que celui des contes de tradition orale.

L'analyse des mondes fictionnels du conte met en évidence un recours massif aux usages négatifs des formes oblatives. On ne peut pas accéder à la logique narrative de nombreux récits en mettant entre parenthèses la face noire du don. La lecture de nos petits récits fait apparaître avec force une tension de la négativité et de la positivité du don, qui est en étroite relation avec la tension narrative elle-même. Se pourrait-il que l'impératif de tenir en haleine les récepteurs induise une accentuation des contrastes du positif et du négatif interactionnel? C'est vraisemblable mais insuffisant comme explication. Car le problème n'est pas seulement quantitatif; il est d'abord et avant tout qualitatif: comment rendre compte des faces ombrées de la réciprocité et de l'unilatéralité et de leurs rapports avec les faces positives?

La sociogenèse de la compréhension apporte sur ce point des éléments intéressants, car elle met en évidence la construction d'espaces de jeu ou espaces de réversibilité virtuelle relativement indéterminés et de plus en plus complexes autour des régularités, des règles et des valeurs. Ces espaces de réversibilité se présentent comme des carrefours de l'action, des ensembles de possibles aux conséquences très diverses que nous traduisons, de façon culturellement variable, en positivité ou en négativité normatives. La spirale de la complexification de la compréhension et de la complexification relationnelle ne peut que déboucher sur des espaces normatifs solidaires des espaces de réversibilité. Tous nos contextes interactifs sont caractérisés par cette dialectique des possibles et de la normativité.

Les mondes du réel et de la fiction expriment certainement de façon différente cette compréhension de l'action, mais ils en dépendent directement tous les deux. 
Ils se construisent sur cette base. C'est leur monde commun. Ce monde commun s'élabore à partir de la représentation; non pas d'une représentation comme miroir mais d'une représentation comme écart, d'un virtuel virtuellement fidèle-infidèle, où les tensions entre justesse, vérité, mensonge, erreur et fiction constituent notre lot incontournable.

Le don n'évoque chez la plupart des sociologues que des dimensions positives. On a tendance à oublier que certaines rhétoriques du don à Dieu, à la patrie et à la famille cachent des fonctions qui ne sont pas que positives, du moins pour certaines catégories d'acteurs. On a tendance aussi à oublier que dans le monde de partage et de don des microcommunautés familiales et amoureuses se déroulent toutes sortes de drames dont les psychologues recueillent les échos. Certes, l'idéal paradisiaque du don positif est plein de promesses; il ne faut pas oublier toutefois que la liberté sur laquelle il repose a pour contrepartie obligatoire un autre demi monde symétrique pour le moins problématique. Et ce n'est qu'en réintégrant cette partie obscure dans l'univers des possibles que le sociologue et l'anthropologue pourront dépasser une position normative naïve et ouvrir sur des compréhensions plus profondes de l'action. La notion de matrice interactive, qui combine contextes interactionnels et possibles offerts par la compréhension de l'action, constitue une proposition dans ce sens.

Institut des sciences sociales et pédagogiques

Université de Lausanne

Andre.Petitat@unil.ch 